\title{
LONG WAVE EFFECTS ON BREAKING WAVES OVER FRINGING REEFS
}

\author{
John T. Goertz ${ }^{1}$, James M. Kaihatu ${ }^{2}$, Alex Sheremet ${ }^{3}$, Ernest R. Smith ${ }^{4}$ and Jane M. Smith ${ }^{5}$
}

\begin{abstract}
Modeling of wave energy transformation and breaking on fringing reefs is inherently difficult due to their unique topography. Prior methods of determining dissipation are based on empirical data from gently sloping beaches and offer only bulk energy dissipation estimates over the entire spectrum. Methods for deducing a frequency dependent dissipation have been limited to hypothesized linkages between dissipation and wave shape in the surf, and have used bulk dissipation models as a constraint on the overall dissipation for mild sloping beaches. However, there is no clear indication that the constraint on the overall level of dissipation is suitable for the entire reef structure. Using these constraints the frequency dependent dissipation rate can be deduced from laboratory data, taken at the Coastal and Hydraulics Laboratory, of wave transformation over reefs. The frequency dependent dissipation rate can then be integrated over the spectrum to derive an empirically-based counterpart to energy flux dissipation. Comparing the bulk energy dissipation estimates for the reef system to the frequency based method allows for the modification of wave breaking parameters in the frequency estimation, to better estimate total dissipation. Since this method is based on the Fourier transform of the time series data, it allows the dissipation to be found as a function of the frequency. This analysis shows that there is a correlation between the amount of energy in the low frequencies of the wave spectrum and certain characteristics of the frequency dependent dissipation coefficient.
\end{abstract}

Keywords: long wave breaking; reef systems; dissipation analysis

\section{INTRODUCTION}

Much of the world's population lives on or near the coast; knowledge of the processes seen in these areas is vital to the infrastructure and safety of the population. Modeling the wave effects near the shoreline is important since waves are the primary driver of nearshore processes such as beach erosion and nearshore circulation. Prediction of wave energy throughout the shoaling and breaking zones is therefore key to determination of these processes in the nearshore.

Accurate modeling of the energy transformation and breaking on fringing reefs is especially difficult due to their unique topography. Many coastal areas that are surrounded by nearshore fringing reefs are prone to lowland flooding events caused by high waves, wave-induced setup and enhanced low-frequency energy generated by breaking on this topography. These effects require close study of breaking processes over steep bathymetry. In addition, reefs do not undergo bathymetric change over the scale of a storm or weather event, and thus cannot evolve to mitigate the destructive effects of a storm in the manner of a sand beach.

\section{Fringing Reefs}

Coral reefs are most common in the South-West Pacific Ocean. Different structure types exist for coral reefs, but one common type is the fringing reef. Many islands are surrounded by these fringing reefs, which grow in shallow water close to the coastline. They are characterized by wide, shallow platforms that sharply drop into deep water on one side, while on the other side going all the way to the shoreline. A cross-sectional view a fringing reef compared to a more common platform reef can be seen in Figure 1.

Fringing reefs protect most coastal areas from wave action by forcing waves to break and dissipate their energy before reaching the shoreline. This forced wave breaking can sometimes cause flooding phenomena in the lowland areas near the shoreline. Some wave and storm conditions, that would not be expected to cause issues along the shoreline, instead cause lowland flooding due to the wave interactions on the shelf of the reef. Significant damage may also occur during typhoons and hurricanes when the wide, shallow reef shelf may cause the surge to build up.

${ }^{1}$ Graduate Research Assistant, Zachry Department of Civil Engineering, Texas A\&M University, 3136 TAMU, College Station, TX 77843-3136, USA, (John.T.Goertz@gmail.com)

${ }^{2}$ Associate Professor, Zachry Department of Civil Engineering , same address as above, (JKaihatu@civil.tamu.edu)

${ }^{3}$ Associate Professor, Department of Civil and Coastal Engineering, University of Florida, 365 Weil Hall, Gainesville, FL 32611, USA, (Alex@coastal.ufl.edu)

${ }^{4}$ Hydraulic Engineer, Coastal and Hydraulics Laboratory, Engineer Research and Development Center, 3909 Halls Ferry Road, Vicksburg, MS, 39180-6199, USA, (Ernest.R.Smith@usace.army.mil)

${ }^{5}$ Hydraulic Engineer, Coastal and Hydraulics Laboratory, same address as above, (Jane.M.Smith@usace.army.mil) 


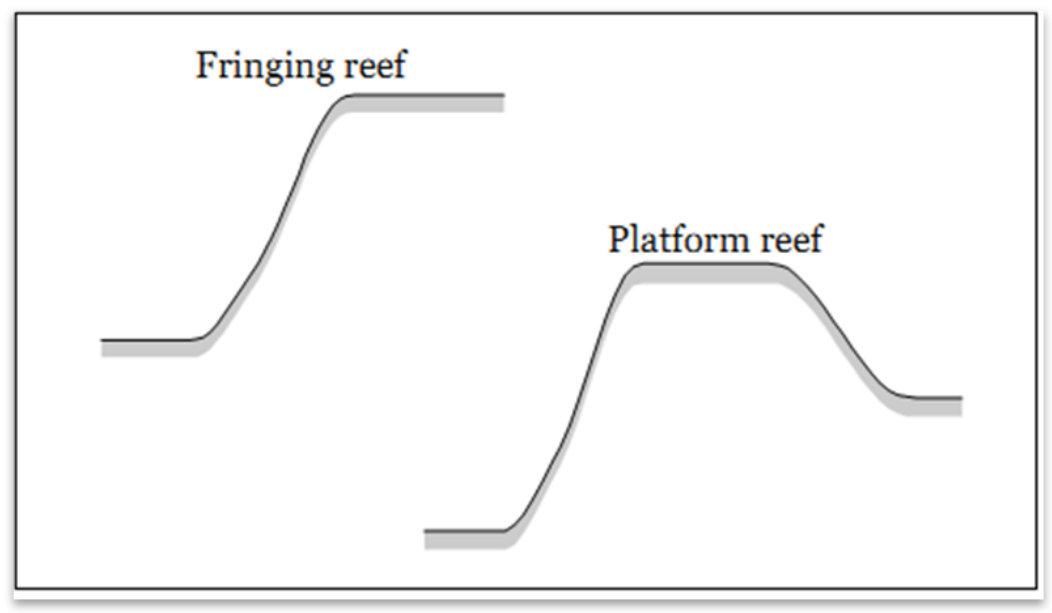

Figure 1: Fringing Reef vs. Platform/Barrier Reef (Source: ERDC/CHL)

\section{Background}

Prior methods of determining energy dissipation, such as Thornton and Guza (1983; hereafter TG83), are based on empirical data from gently sloping beaches and offer only bulk energy dissipation estimates over the entire spectrum. There is no clear indication that the constraint on the overall level of dissipation from TG83 is suitable for the entire reef structure due to the steep slope at the toe of the reef. Janssen and Battjes (2007; hereafter JB07) offers a method for breaking wave dissipation over steep slopes.

It is possible to investigate the breaking process at levels deeper than bulk dissipation estimates. For example, Kaihatu and Kirby (1995) developed a frequency-dependent dissipation mechanism incorporated into a nonlinear wave model. While the overall dissipation (integrated over frequencies) was constrained by bulk estimates, the inter-frequency variation of dissipation allowed this process to be responsive to the nonlinear evolution of irregular waves through the shoaling and surf zones. Theoretical arguments for frequency-dependent dissipation were made by Kirby and Kaihatu (1996; hereafter KK96) and augmented with further data analysis by Kaihatu et al (2007). Furthermore, KK96 formulated instantaneous dissipation estimates based on eddy viscosity-based formulations of dissipation (Zelt 1991) triggered by the slope of the forward face of the wave. These can be analyzed to obtain distributions of dissipation over the frequency range of the spectrum and compared to hypothesized distributions.

The resulting frequency dependent dissipation rate can be integrated over the spectrum (Kaihatu and El Safty 2011; hereafter KE11) to derive a bulk dissipation estimate that is comparable to the previously mentioned empirically-based methods but which are instead based on a population of instantaneous dissipation events rather than assumed probability distributions. With suitable adjustment of breaking parameters through comparison to bulk models, the result offers both the proper energy decay rate over the steep reef face and a view of the individual breaking waves.

\section{LABORATORY EXPERIMENTS}

\section{SWIMS 2D Reef Experiment}

The data set to be used for the dissipation analysis comes from a laboratory experiment performed by the Surge and Wave Island Modeling Studies (SWIMS) program at the Engineering Research and Development Center, Coastal and Hydraulics Laboratory, in the fall of 2010. The data set is comprised of random wave groups over a prototype fringing reef in a $2 \mathrm{D}$ wave flume. The layout of the experiment is shown in Figure 2.

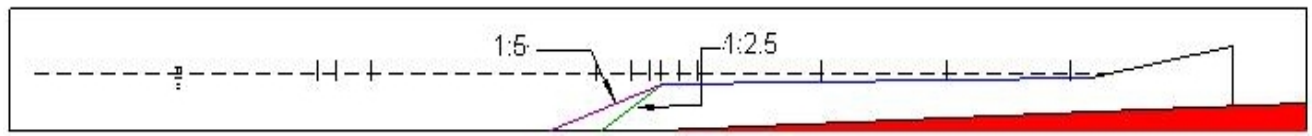

Figure 2: Wave Flume Layout for SWIMS 2d Reef Experiment 
The experiment was conducted in a 3-ft wide flume with a 1:50 model to prototype scale reef. The modeled reef consists of either a 1:2.5 or a 1:5 reef slope and a 24-ft long reef section at a slope of 1:100 that is constructed of molded acrylic to represent reef bathymetry. The goal of the experiment was to collect wave and run-up data over a range of wave heights and periods, water levels, and reef roughness. Forty-three design wave conditions were run for each model slope. Twelve gages were placed along the flume to measure water surface elevation and wave heights.

This data set is chosen for this study for several reasons. First and foremost is the fact it attempts to model the wave effects for a fringing reef. The acrylic reef top is modeled after real world data scans of a reef in the south pacific. This experiment also attempts to recreate the natural roughness of a reef by creating a rough top for the acrylic reef. This experiment also contains a dense gage concentration at the top of the steep slope, where the wave energy undergoes a high rate of evolution. Other similar experiments use sparser gage distributions and thus do not capture this rapid evolution near the crest.

\section{BULK DISSIPATION ANALYSIS}

\section{Thornton and Guza 1983}

Thornton and Guza 1983, an extension of Battjes and Janssen (1978), offers a method of estimating bulk dissipation for random wave trains. This is accomplished through use of a Rayleigh distribution to represent wave height distribution through the surf zone. Based on empirical data, a modified and weighted distribution is used that limits the upper wave heights.

The energy dissipation rate of the wave is modeled after a periodic bore and, after applying the modified and weighted Rayleigh distribution, results in the bulk dissipation estimation:

$$
\left\langle\varepsilon_{b}\right\rangle=\frac{3 \sqrt{\pi}}{16} \rho g B^{3} \frac{H_{R M S}^{5}}{\gamma^{2} h^{3}}\left[1-\frac{1}{\left(1+\left(H_{R M S} / \gamma h\right)^{2}\right)^{5 / 2}}\right]
$$

Here $\varepsilon_{b}$ is the bulk dissipation, $\rho$ is the fluid density, $g$ is acceleration due to gravity, $\bar{f}$ is the median frequency of the wave series, $H_{R M S}$ is the root-mean-square wave height, and $h$ is the depth at the gage location. The variables $B$ and $\gamma$ are free parameters and are found by fitting the value to the measured root-mean-square wave height using TG83.

From these estimates of the root-mean-square wave height, shown in Figure 3, it became evident that the model is a poor match with the values calculated from the experimental data on the steep slope portion of the reef. The wave heights were underestimated due to the truncation of higher wave heights that are more representative of waves traversing a mild sloping beach, but do not properly model the effects on a steep slope.

\section{Janssen and Battjes 2007}

For steep-sloped beaches and areas where the surf zone is fully saturated, the empirically-based weightings for the wave height distribution discussed by TG83 may be insufficient to adequately describe the portion of waves in the distribution higher than the saturation cutoff, since steep slopes may allow waves to shoal beyond their breaking limit. A model for this is proposed by Janssen and Battjes (2007). Rather than empirical weightings, a full Rayleigh distribution in concert with a single incipient breaking wave height is used. This wave height cutoff is not representative of a saturated surf zone (i.e., some fraction of the water depth), but is instead a function of the offshore wave steepness.

To account for the difference in slope and water level, a different modified and weighted Rayleigh distribution is used and integrated with respect to a breaker height $H_{b}$, above which the wave is assumed to be breaking. The form of this weighted distribution allows waves to break that are smaller than the reference breaker height. After applying this distribution, the new form of the bulk dissipation is expressed as with $R=H_{b} / H_{R M S}$. The term erf refers to the error function, which is described in detail in Abramowitz and Stegun (1965):

$$
\langle\varepsilon\rangle=\frac{3 \sqrt{\pi}}{16} B \bar{f} \rho g \frac{H_{R M S}^{3}}{h}\left[1+\frac{4}{3 \sqrt{\pi}}\left(R^{3}+\frac{3}{2} R\right) \exp \left[-R^{2}\right]-\operatorname{erf}(R)\right]
$$

From this, JB07 goes on to find an estimate of root-mean-square wave height. Comparison of this root-mean-square estimate is found to be consistent with those of the data on the steep slope portion of 
the reef. However, on the mild slope top of the reef the model returns values that are not physically possible as seen in Figure 3. From this we can infer (due to the root-mean-square estimations basis on the dissipation expression) that the model will be able to estimate dissipation on the steep slope portion of the reef but not the mild slope portion.

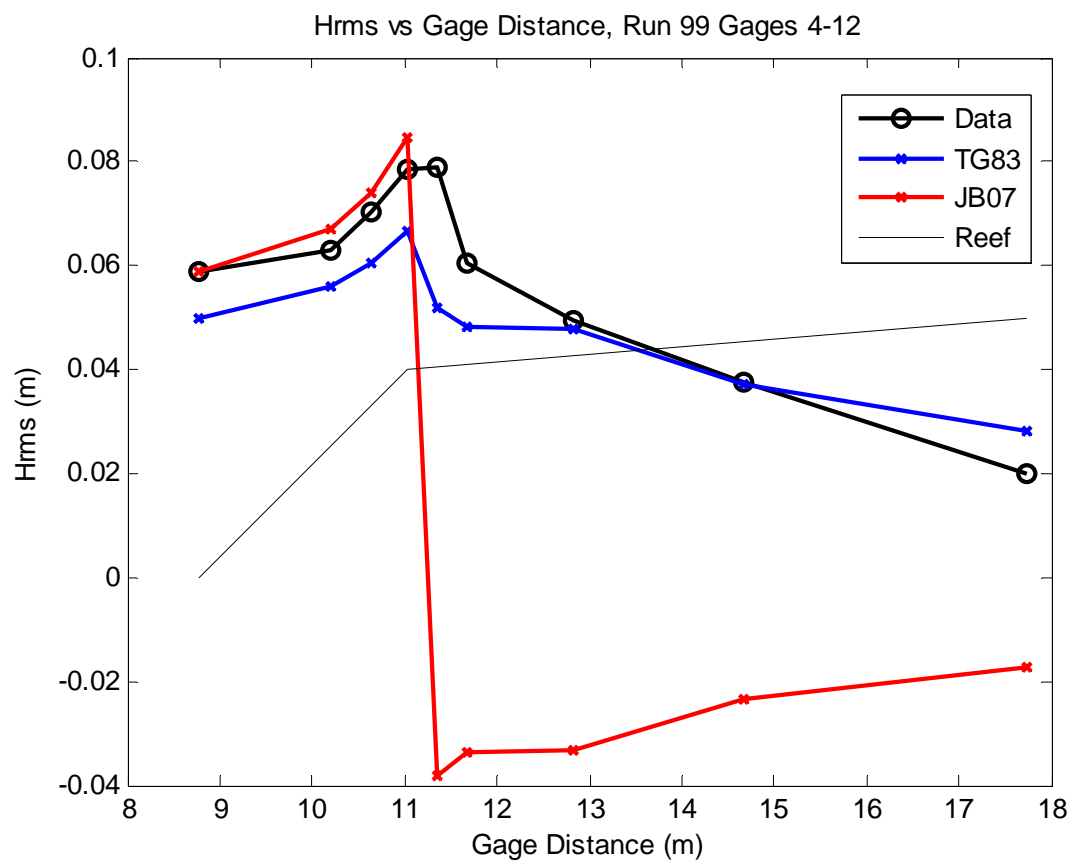

Figure 3: Hrms vs Gage Location. Comparing calculated Hrms from data to TG83 and JB07 models

Model Combination

Since neither model can be used to effectively estimate the wave height over the entire reef system, it can be expected that this holds true for the bulk dissipation estimation as well. In order to derive a total bulk dissipation estimate over the whole reef system, a combination of the two models is utilized. The dissipation model of JB07 will be used for the steep portion of the reef while the model of TG83 will be used for the mild slope portion of the reef. The zones where each model is used can be seen in Figure 4.

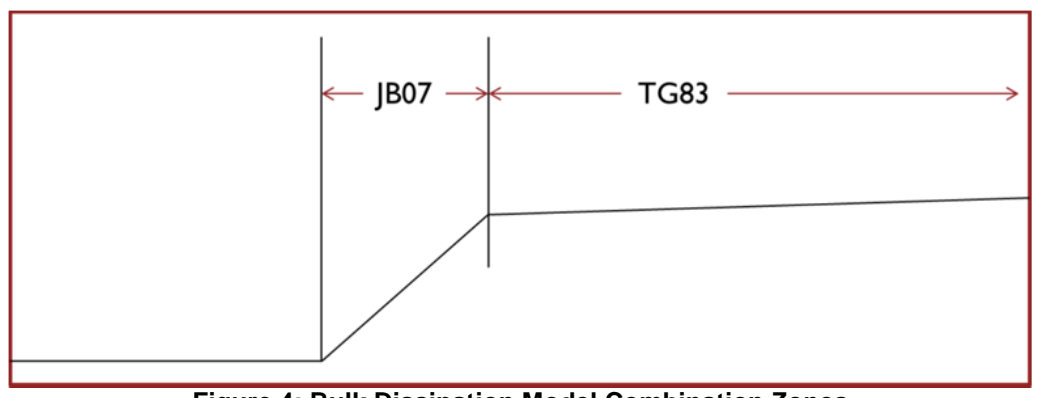

Figure 4: Bulk Dissipation Model Combination Zones

The result of using these two models in their region of best estimation is one model that can estimate the root-mean-square wave height that is a good match to the experimental data, which can be seen in Figure 5. This in turn gives us good dissipation estimates for the complex reef system. However, the model still allows only bulk dissipation estimates, with no further insight into the dissipation characteristics over the steep slope. We require a method for investigating the dissipation at a finer scale, using the integrated dissipation as verification metric. 


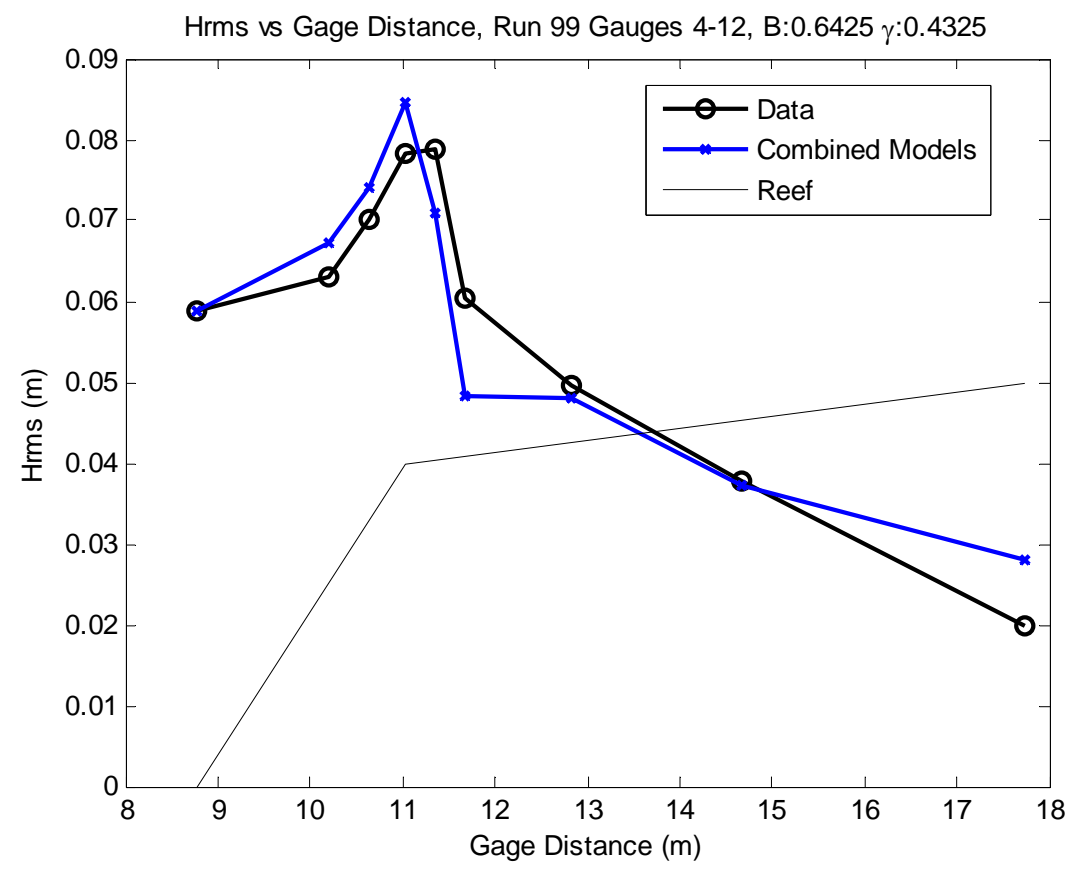

Figure 5: Hrms vs. Gage Location. Comparing calculated Hrms from data to combined models.

\section{FREQUENCY-BASED DISSIPATION ANALYSIS}

\section{Rate Calculation}

Instead of assuming overall characteristics of the spectrum, Kirby and Kaihatu (1996) use a timedependent description of instantaneous wave breaking in order to analyze dissipation characteristics over a random wave train. The instantaneous dissipation is based on a transformed version of the eddy viscosity model of Zelt (1991):

$$
\begin{gathered}
\varepsilon=-\rho\left(\frac{\eta}{h}\right)\left(v_{b} \eta_{t}\right)_{t} \\
v_{b}=B_{*} \gamma_{*}^{2} h \eta_{t}
\end{gathered}
$$

Here $\varepsilon$ is the time dependent dissipation, $\rho$ is the fluid density, $\eta$ is the free surface time series, and $h$ is the depth at the gage location. The variables $B *$ and $\gamma_{*}$ are not the same free parameters from the bulk dissipation models. For this equation $B_{*}$, based on $\eta_{t}$, is a value between zero and one that represents whether or not the wave is breaking. The variable $\gamma_{*}$ is a mixing length parameter. The subscript $t$ in the equation represents a partial derivative taken with respect to time. The result can be converted from a dissipation time series to spectral densities of dissipation and using this along with the smoothed spectral density of the complex amplitudes, KK96 estimate the dissipation rate $\alpha_{n}$ as:

$$
\begin{gathered}
\alpha_{n}=\frac{1}{2 C_{g n} \sqrt{2 \Delta f}} \frac{1}{\rho g} \frac{\sqrt{S_{\varepsilon}\left(f_{n}\right)}}{S_{\eta}\left(f_{n}\right)} \\
S_{\varepsilon}(n)=\frac{\left\langle\left|\varepsilon_{b n}\right|^{2}\right\rangle}{2 \Delta f} \\
S_{\eta}(n)=\frac{\left\langle\left|A_{n}\right|^{2}\right\rangle}{2 \Delta f}
\end{gathered}
$$


Here we find that $\varepsilon_{b n}$ is the Fourier transform of the instantaneous dissipation, $\varepsilon$, computed directly from measured data, $A_{n}$ is the complex amplitude of the time series of free surface elevations, $\eta$. Using this process allows the time series information to be used in estimating the dissipation rate independent of any assumptions concerning probability distributions.

\section{Bulk Dissipation}

Bulk dissipation is generally represented as a change in energy flux, such as in TG83 and JB07. However, these parametric formulations not only differ based on the exact weighting function used for the probability distribution, but are also dependent on several free parameters that must be fit to data. A more detailed and robust model for estimating bulk dissipation offers more insight into the nature of energy dissipation. Kaihatu and El Safty (2011) formulated a bulk dissipation mechanism based on a summation of the detailed dissipation rate estimations, as seen below:

$$
-D=-\rho g \sum_{n=1}^{N} C_{g n}\left|A_{n}\right|^{2} \alpha_{n}
$$

By summing over the whole spectrum of dissipation rates in the frequency domain they arrived at an estimate of bulk dissipation without the a priori assumption of a probability distribution.

We now apply the dissipation rate and bulk dissipation estimate to the data from the SWIMS 2D reef experiment. When this is compared to model values from the probabilistic method, we find that the frequency based method overestimates the total dissipation for each gage location. This can be seen in Figure 6. This is likely due to the fact that the instantaneous dissipation is a function of the inverse of the free surface time series spectrum. Since this spectral energy density is small at high frequencies, alpha can become very large.

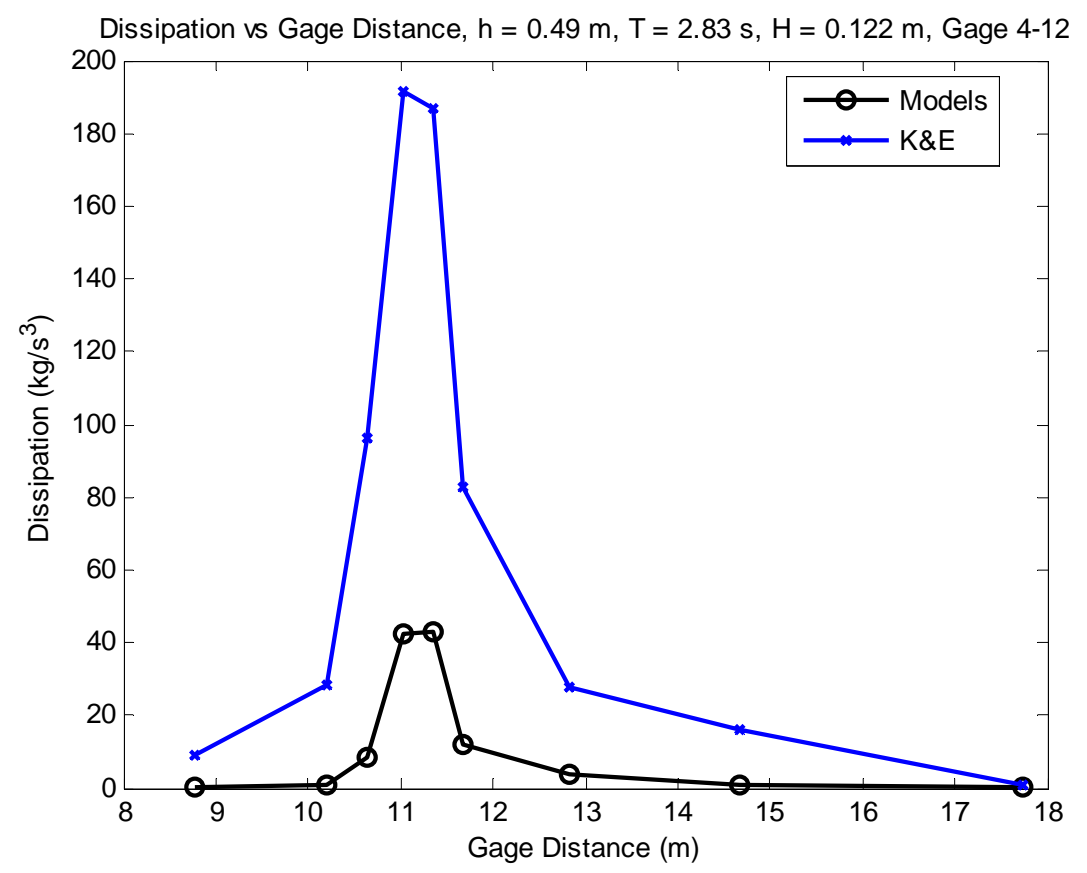

Figure 6: Frequency and Probabilistic Based Dissipation vs. Gage Distance. Run \#102 with depth $=0.49 \mathrm{~m}$ and incident wave characteristics of period $=2.83 \mathrm{~s}$ and wave height $=0.122 \mathrm{~m}$.

\section{Dissipation Constraint}

The frequency-based bulk dissipation must be constrained in order to account for the overestimation. To accomplish this, the breaking parameter, $B_{*}$, in the Zelt (1991) eddy viscosity model is modified. A constant value in this parameter was derived based on a solitary wave breaking over a mild sloping beach. The value is based on a ratio of breaking wave height to depth in order to replicate the breaking of a solitary wave. 
Use of the solitary wave estimate impacts the overestimate of dissipation because the data set consists of random waves, which have different wave breaking characteristics. Modification of the constant value in the breaking parameter alters the breaking criterion and, consequently, the number of waves considered to be breaking. An increase in the value of this parameter reduces the overall number of breaking events and decreases the bulk dissipation. The effect of this change can be seen in Figure 7.

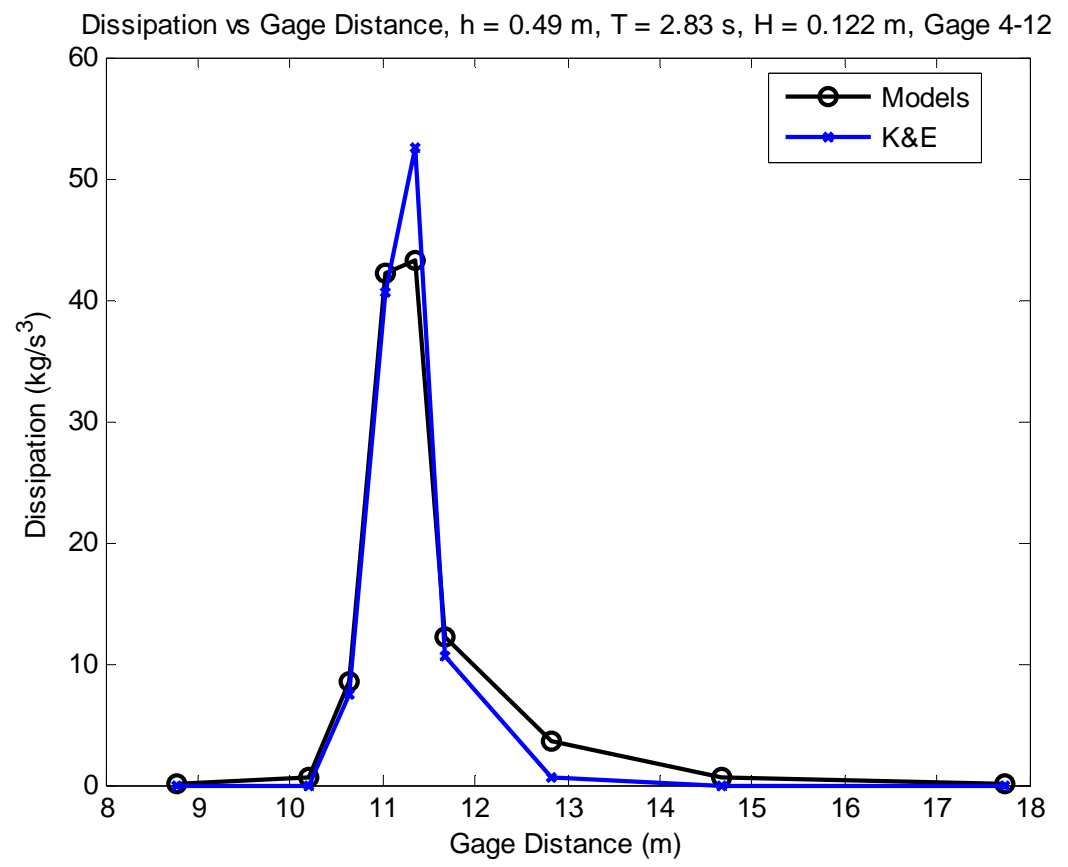

Figure 7: Frequency and Probabilistic Based Dissipation vs. Gage Distance. Constant value in breaking parameter tuned to larger value in order to limit number of breaking waves.

By appropriate tuning of this parameter, the amount of dissipation at the most energetic wave breaking locations are in agreement with those of the probabilistic models. However, the dissipation estimates at the gages further up the mild slope of the reef are under predicted. Despite this fact, with the result of the estimation at the portion of the reef that has the greatest energy and highest dissipation gives us results that agree with those expected by the combined probabilistic models.

\section{CONLUSIONS}

In this study we investigate the use of probabilistic bulk dissipation models for use on a reef system, and found that no one model accurately estimates dissipation over the entire reef structure. Instead a combination of two models must be used, one for the steep portion, and one for the mild slope reef top. This combined model analysis is limited by its reliance on wave height distribution model weightings, and its inability to define finer detail than the bulk dissipation.

We then investigated the use of a frequency-based method of dissipation calculation that is based on a combination of the spectrum of the time series data to calculate the dissipation rate for the system. The overall bulk dissipation can then be determined from this dissipation rate. Using the combined probabilistic models for the reef system as a metric for dissipation, we are able to compare these estimates to those from the frequency-based analysis, and find that the frequency-based method overestimates the total dissipation in the system. Through further use of the probabilistic models we are then able to modify the eddy viscosity breaking parameter to account for the breaking of random waves.

The frequency-based analysis allows for the estimation of dissipation in a region when all that is known is the time series free surface elevation data and the depth, with no further reliance on 
probability distributions and corresponding weightings. It also allows for analysis of wave energy effects in finer detail than bulk dissipation models allow.

Future work in this area will involve analysis and parameterization of the dissipation characteristics at levels of detail finer than normally allowed in bulk models. Further study is also to be performed into developing probability distribution functions of the instantaneous dissipation rates.

\section{ACKNOWLEDGMENTS}

Permission to publish this work was granted by the Office, Chief of Engineers, US Army Corps of Engineers. This work was conducted under the Surge and Wave Island Modeling Studies under the Coastal Field Data Collection Program of the Coastal and Hydraulics Laboratory, US Army Engineer Research and Development Center.

\section{REFERENCES}

Abramowitz, M., and I. Stegun. 1965. Handbook of Mathematical Functions, Dover, New York, NY, $1045 p$.

Battjes, J.A., and J.P.F.M. Janssen. 1978. Energy loss and set-up due to breaking of random waves, Proceedings of $14^{\text {th }}$ International Conference on Coastal Engineering, ASCE, 466-480.

Janssen, T.T., and J.A. Battjes. 2007. A Note on Wave energy Dissipation Over Steep Beaches, Coastal Engineering, 54, 711-716.

Kaihatu, J.M., and Kirby, J.T. 1995. Nonlinear transformation of waves in finite water depth. Physics of Fluids, 7, 1903-1914.

Kaihatu, J.M., J. Veeramony, K. L. Edwards, and J.T. Kirby. 2007. Asymptotic behavior of frequency and wave number spectra of nearshore shoaling and breaking waves. Journal of Geophysical Research, 112, doi: 10.1029/2006JC003817.

Kaihatu, J.M., and H.M. El Safty. 2011. Spectral Description of Energy Dissipation in Breaking Wave Groups, Proceedings of $32^{\text {nd }}$ International Conference on Coastal Engineering : Waves 19.

Kirby, J.T., and J.M. Kaihatu. 1996. Structure of frequency domain models for random wave breaking, Proceedings of 25th International Conference on Coastal Engineering, ASCE, 1144-1155.

Thornton, E.B., and R.T. Guza. 1983. Transformation of Wave Height Distribution, Journal of Geophysical Research, 88, 5925-5938.

Zelt, J.A. 1991. The run-up of nonbreaking and breaking solitary waves. Coastal Engineering, 15,205246. 